\title{
DA COR À COR INEXISTENTE: UMA REFLEXÃO SOBRE ESPECTROS ELETRÔNICOS E EFEITOS CROMÁTICOS
}

\author{
Henrique E. Toma* e Leonardo da Silva Bonifácio
}

Instituto de Química, Universidade de São Paulo, CP 26077, 05513-970 São Paulo - SP

Fauze J. Anaissi

Departamento de Química, Universidade Estadual do Centro-Oeste, CP 3010, 85040-080 Guarapuava - PR

Recebido em 21/6/04; aceito em 4/1/05; publicado na web em 13/4/05

\begin{abstract}
FROM COLORS TO INEXISTENT COLORS: A REFLECTION ON ELECTRONIC SPECTRA AND CHROMATIC EFFECTS. Inexistent colors have been the inspiring theme of investigation by Israel Pedrosa, a Brazilian artist, who has devoted his life to creative painting, exploiting the chemical and physical effects associated with light, especially those generated in the light refraction domain. In this article, by focusing on the electronic spectra of phthalocyanines and gold nanoparticles, we discuss how such effects can influence the spectroscopic measurements, leading to inexistent bands and transitions.
\end{abstract}

Key words: inexistent colors; refraction colors; Israel Pedrosa.

\section{INTRODUÇÃO}

Com este estranho título "Da Cor à Cor Inexistente", queremos reverenciar o mestre Israel Pedrosa ${ }^{1}$ o mais intrigante dos artistas da cor, e sua obra, tão bem retratada nas palavras de Jacob Klintowitz": "Não satisfeito com a maestria de seu aprendizado, Israel Pedrosa dedicou-se a pesquisar a refração cromática e estudou a maneira de conduzir esta refração até torná-la visível. O artista, utilizando o pigmento (cor química) e a refração da cor (cor física), construiu uma obra extremamente rica de implicações culturais e realizou o seu mais velho sonho, o de pintar com a luz. As suas formas são estabelecidas em espaços determinados, a estrutura da forma é geométrica, a composição é sólida e, várias vezes, clássica, e o resultado é inovador. Ao domínio da refração cromática o artista denominou Domínio da Cor Inexistente. É esta inexistência, plena de vida poética e reveladora de novos horizontes cromáticos, a essência de seu trabalho e razão maior do encantamento de sua produção. A realidade é constituída e organizada com a mais sutil das referências e o além da cor torna-se cor diante dos nossos olhos."

Quebrando este encantamento poético, poderíamos indagar se a refração, que engana tão bem os nossos olhos, também afetaria as cores espectrais captadas por um espectrofotômetro. Ao abordar esse tema ${ }^{3}$, nosso objetivo foi chamar a atenção para os aspectos diferenciais associados às várias formas de monitoração espectroscópica (i.e., absorção, transmissão, reflexão), levando em conta também os efeitos peculiares relacionados com o estado físico (i.e., sólidos, nanopartículas, filmes e soluções).

Como objeto de estudo, foi escolhida a ftalocianina de cobre, por ser a representante típica da geração moderna de pigmentos utilizados nas indústrias automobilística e eletrônica e nas artes ${ }^{4}$. $\mathrm{Na}$ realidade optou-se pelo derivado tetrassulfonado, por ser solúvel em água e permitir a formação de filmes moleculares por simples evaporação da solução. Deve ser esclarecido, porém, que mais do que simples pigmentos, as ftalocianinas apresentam propriedades ópticas, eletrônicas, eletroquímicas e catalíticas bastante espe-

*e-mail: henetoma@iq.usp.br ciais. Sua importância vem crescendo exponencialmente neste limiar da era nanotecnológica, participando cada vez mais do design de dispositivos sensoriais e de eletrônica molecular. As ftalocianinas e seus metaloderivados (Figura 1) constituem moléculas planas, simétricas. Seus cristais moleculares são brilhantes e, às vezes, metálicos. Suas altas estabilidades térmica e química favorecem a utilização tecnológica, principalmente sob a forma de filmes finos.

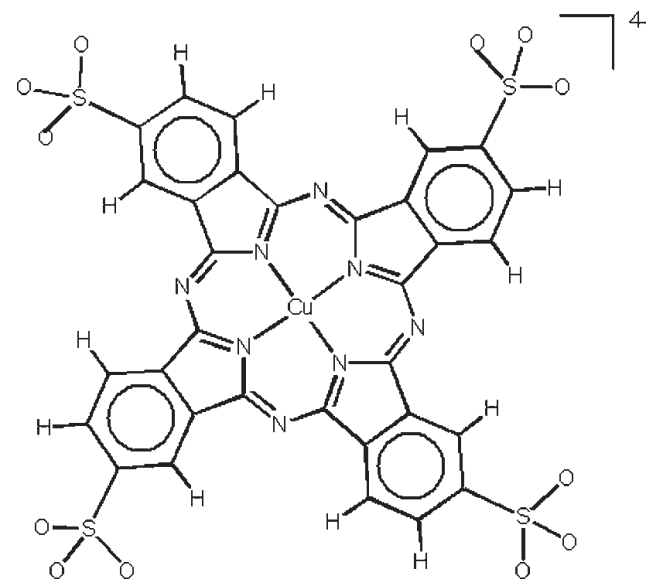

Figura 1. Representação estrutural da ftalocianina de cobre tetrassulfonada

Outro exemplo explorado foram as nanopartículas de ouro, que têm ganhado importância crescente especialmente em estudos inspirados na abordagem "bottom-up" das nanociências ${ }^{5}$. A razão para tal fato emerge de suas propriedades ópticas e eletrônicas. Nelas, os elétrons superficiais (plasmons) estão distribuídos em estruturas de bandas com energias distintas. Desse modo a energia de transição eletrônica, ou seja, a cor das nanopartículas depende da densidade de estados eletrônicos e do tamanho das nanopartículas. Além disso, devido a seu caráter mole (soft) o ouro é capaz de ligar-se a grupamentos tióis, permitindo o desenvolvimento de sistemas metalorgânicos de alta complexidade. Aliando tal propriedade a suas características optoeletrônicas, abre-se um amplo leque de possibilidades de aplicação em nanotecnologia molecular, principalmente em sensores químicos e biológicos. 


\section{A REFRAÇÃO CROMÁTICA E O DOMÍNIO DA COR INEXISTENTE}

Para entender a refração cromática, é importante analisar como a luz perturba os elétrons em uma molécula ${ }^{6,7}$.

A luz tem um campo elétrico $\mathbf{E}$ que interage com as cargas elétricas (q) em um material, produzindo uma força $\mathbf{F}=$ q.E. Essa força acaba deslocando a densidade eletrônica em relação aos núcleos atômicos, provocando uma separação de carga e dando origem a um dipolo induzido, sendo $\mu$ o seu momento.

A facilidade com que os elétrons se deslocam sob ação do campo elétrico é expressa pela polarizabilidade, $\alpha$. Assim,

$\boldsymbol{\mu}=\alpha . \mathbf{E}$

Deve ser notado que o campo elétrico associado a uma radiação oscila com uma freqüência $\omega$, sendo representado por $\mathbf{E}(\omega)$. Se a resposta dos elétrons for instantânea, a polarização induzida irá ter a mesma frequiência $(\omega)$ e fase que a radiação. Por outro lado, $\boldsymbol{\mu}$ e $\mathbf{E}$ são quantidades vetoriais, apresentando direção além de grandeza numérica.

Se os átomos nas moléculas são polarizados de forma independente pela luz, sem que existam acoplamentos, a polarizabilidade resultante é dada pela soma das polarizabilidades atômicas, isto é $\chi(\omega)=\Sigma \alpha(\omega)$. Dessa forma, a polarizabilidade de um material passa a ser expresso por

$\mathbf{P}=\boldsymbol{\chi}(\omega) \mathbf{E}(\omega)$

Devido ao deslocamento de cargas eletrônicas, o campo elétrico total (D) no interior do material é dado pela soma do campo elétrico externo, $\mathbf{E}$, e do campo elétrico induzido, $\mathbf{I}=4 \pi \mathbf{P}$, isto é:

$\mathbf{D}=\mathbf{E}+4 \pi \mathbf{P}=(1+4 \pi \chi) \mathbf{E}$

É interessante notar que sendo $\chi$ uma grandeza vetorial, a polarização induzida pode levar a uma orientação do campo elétrico interno (D) diferente daquela do campo aplicado (E).

Na prática, entretanto, os dois parâmetros mais usados para caracterizar a polarização de um material, são a constante dielétrica

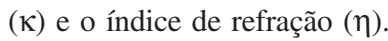

A constante dielétrica em uma dada direção é definida como a razão entre o campo elétrico no interior do material, $\mathbf{D}$, e o campo aplicado, $\mathbf{E}$, isto é:

$\kappa=\mathbf{D} / \mathbf{E}=1+4 \pi \chi$

O índice de refração é igual à razão entre a velocidade da luz (v) através do material, e no vácuo (c), isto é, $\eta=$ c/v, e está relacionado com a constante dielétrica através da equação de Maxwell:

$\kappa=\eta^{2}$

Como já foi mencionado, na passagem da luz, as nuvens eletrônicas podem responder instantaneamente aos campos elétricos oscilantes, sem que se altere a velocidade de propagação. Entretanto, quando isso não acontece, os campos oscilantes e induzidos ficam defasados no espaço e no tempo e, em consequiência, a velocidade de propagação é reduzida, observando-se o fenômeno da refração.

Quando o material também é um absorvedor de luz, esse efeito é ainda mais pronunciado, e a refração passa a incorporar mais um termo k, imaginário, sendo expresso pela refração total $(\mathrm{N})$ :
$\mathrm{N}=\eta+\mathrm{i} . \mathbf{k}$

O termo $\mathbf{k}$ atinge valor máximo quando ocorrem transições eletrônicas do estado fundamental para estados excitados, e relaciona-se diretamente com a força do oscilador (f) ou com coeficiente de absorção (extinção), $\varepsilon$ ( $\mathrm{mol}^{-1} \mathrm{~L} \mathrm{~cm}^{-1}$ )

$\mathbf{k}=(1 / 4 \pi) 2,303 \lambda \varepsilon c$

onde $\lambda$ é o comprimento de onda, e c a concentração da espécie absorvedora $\left(\mathrm{mol} \mathrm{L}^{-1}\right)$.

Um caso muito especial é o da reflexão especular, observada por ex. quando a luz incide sobre superfícies metálicas ou vários tipos de filmes moleculares. Para representar esse fenômeno, utiliza-se o coeficiente de reflexão, ou refletividade, $R$, que corresponde à relação entre a intensidade do feixe refletido e a do feixe incidente, atingindo valor máximo quando a incidência ocorre perpendicularmente à superfície.

Nesse caso, pela equação de Maxwell, R passa a ser dado por ${ }^{6}$ :

$\mathrm{R}=[(\mathrm{N}-1) /(\mathrm{N}+1)]^{2}$

Substituindo pela expressão de $\mathrm{N}$ e depois multiplicando pelo complexo conjugado

$R=\left[\left(\mathbf{k}^{2}+\eta^{2}+1\right)-2 \eta\right] /\left[\left(\mathbf{k}^{2}+\eta^{2}+1\right)+2 \eta\right]$

Essa expressão mostra que a refletividade tende a 1 quando o coeficiente de absorção for muito maior que o índice de refração, i.e. $\mathbf{k}>>$ ๆ. Tal situação caracteriza a refletividade metálica.

Para a maioria dos metais, $\mathbf{k}$ e $\eta$ não mudam muito, e a refletividade praticamente não depende do comprimento de onda. Para metais coloridos, como cobre e ouro, em concordância com a Equação 9, observa-se que a refletividade é máxima justamente nos comprimentos de onda onde ocorre maior absorção, ou seja próximos do vermelho ou amarelo, respectivamente.

Finalmente, para lidar com a complexidade das cores, é importante lembrar que os materiais, ao absorverem luz branca, podem adquirir a tonalidade dada pelas cores complementares justamente que não foram absorvidas (mecanismo de subtração), ou então, de forma oposta, pela intensificação da refletividade nos comprimentos de onda de máxima absorção, conforme expressa pela Equação 9. Esses dois mecanismos estão esquematizados na Figura 2.

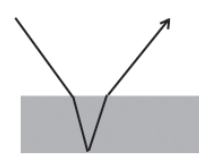

a

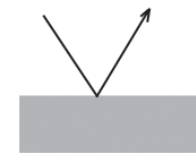

b
Figura 2. Produção de cor através da: a) entrada, seguida pela absorção da luz, e saída dos componentes não absorvidos (mecanismo de subtração), predominando as cores complementares;: b) intensificação da refletividade superficial, predominando a cor absorvida (Equação 9)

\section{ESTUDO DE CASO: AS FTALOCIANINAS DE COBRE}

\section{Fotomicrografias ópticas por transmissão ou reflectância}

Na Figura 3 são mostradas imagens obtidas para a ftalocianina de cobre. A imagem à esquerda, mostra uma foto obtida através de 
fotografia digital, do material depositado sobre uma placa de vidro, a partir da evaporação de uma solução aquosa, onde se destaca a coloração azul intensa, característica da espécie. Nas demais imagens são mostradas fotomicrografias do filme, obtidas por reflectância especular (imagem central) e a por transmissão (imagem à direita). Note que, por reflectância especular o filme apresenta coloração avermelhada, enquanto que por transmissão, a coloração é azul.

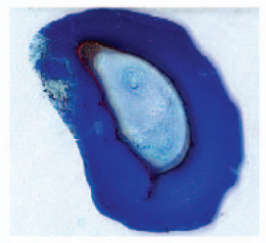

a

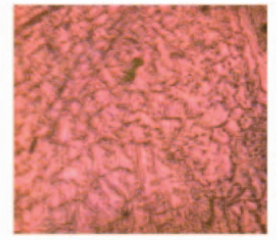

b

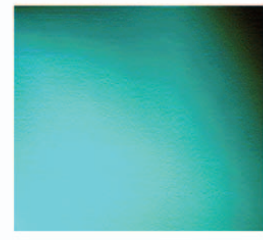

C
Figura 3. Fotografia digital de um filme de ftalocianina de cobre sobre vidro (à esquerda), e micrografias ópticas obtidas por reflectância especular (ao centro) e por transmissão (à direita)

\section{Espectros Eletrônicos}

Na Figura 4 estão registrados os espectros eletrônicos da ftalocianina de cobre tetrassulfonada, em solução, sob a forma de pó, e de filme, por transmissão e reflexão.

Os espectros eletrônicos das porfirinas e ftalocianinas são caracterizadas por bandas intensas na região entre $600-800 \mathrm{~nm}$, conhecidas pela sigla $Q$, e uma outra banda na região entre $350-450$ $\mathrm{nm}$, denominada banda $\mathrm{B}$ ou Soret. Devido à planaridade das moléculas e ao elevado grau de conjugação eletrônica, é bastante comum a ocorrência de interações hidrofóbicas entre os anéis, favorecendo a associação molecular em solução e no estado sólido. Esse efeito provoca um desdobramento das bandas Q, com deslocamentos que têm sido interpretados através de um modelo de acoplamento de excitons ${ }^{8,9}$. De modo geral, as espécies monoméricas apresentam bandas $\mathrm{Q}$ mais finas, ao passo que as bandas mais largas indicam espécies associadas.

Em solução a ftalocianina (Figura 4a) apresenta uma banda Soret em $338 \mathrm{~nm}$ e a banda Q aparece desdobrada, devido à ocorrência de espécies monoméricas $(669 \mathrm{~nm})$ e diméricas $(630,588)$ No espectro do pó (4b) observa-se um alargamento das bandas, indicativo da intensificação das interações intermoleculares, preservando, entretanto, a mesma faixa espectral e, portanto, a coloração das amostras.

Diferenças significativas são observadas nos espectros do filme. Na Figura 4c, o espectro foi obtido por transmissão, e apresenta uma banda Q bastante larga. No processo de formação de filme, as moléculas de ftalocianina formam empilhamentos que são mantidos por forças hidrofóbicas, levando a uma extensão dos acoplamentos eletrônicos e ao alargamento da banda. Na Figura 4d, o espectro foi obtido por reflectância especular, revelando mudanças drásticas, em relação ao espectro de transmissão. A superposição dos espectros das Figuras 4c e 4d demonstra que no máximo de absorção pelo filme, medido por transmissão, tem-se um mínimo correspondente no espectro de reflexão especular. Conforme previsto pela Equação 9, nessa região de alta absorção as ftalocianinas comportam-se como refletores metálicos, i. é, refletem mais intensamente em comprimentos de onda em que mais absorvem. Fora dessa região, os mecanismos de absorção (subtração) e reflexão (soma) combinam-se, gerando picos aparentes em 556 e $700 \mathrm{~nm}$ que, na realidade, não existem. Tem-se assim, uma medida espectroscópica da cor real (Figura 4c) e da "cor inexistente" (Figura 4d).

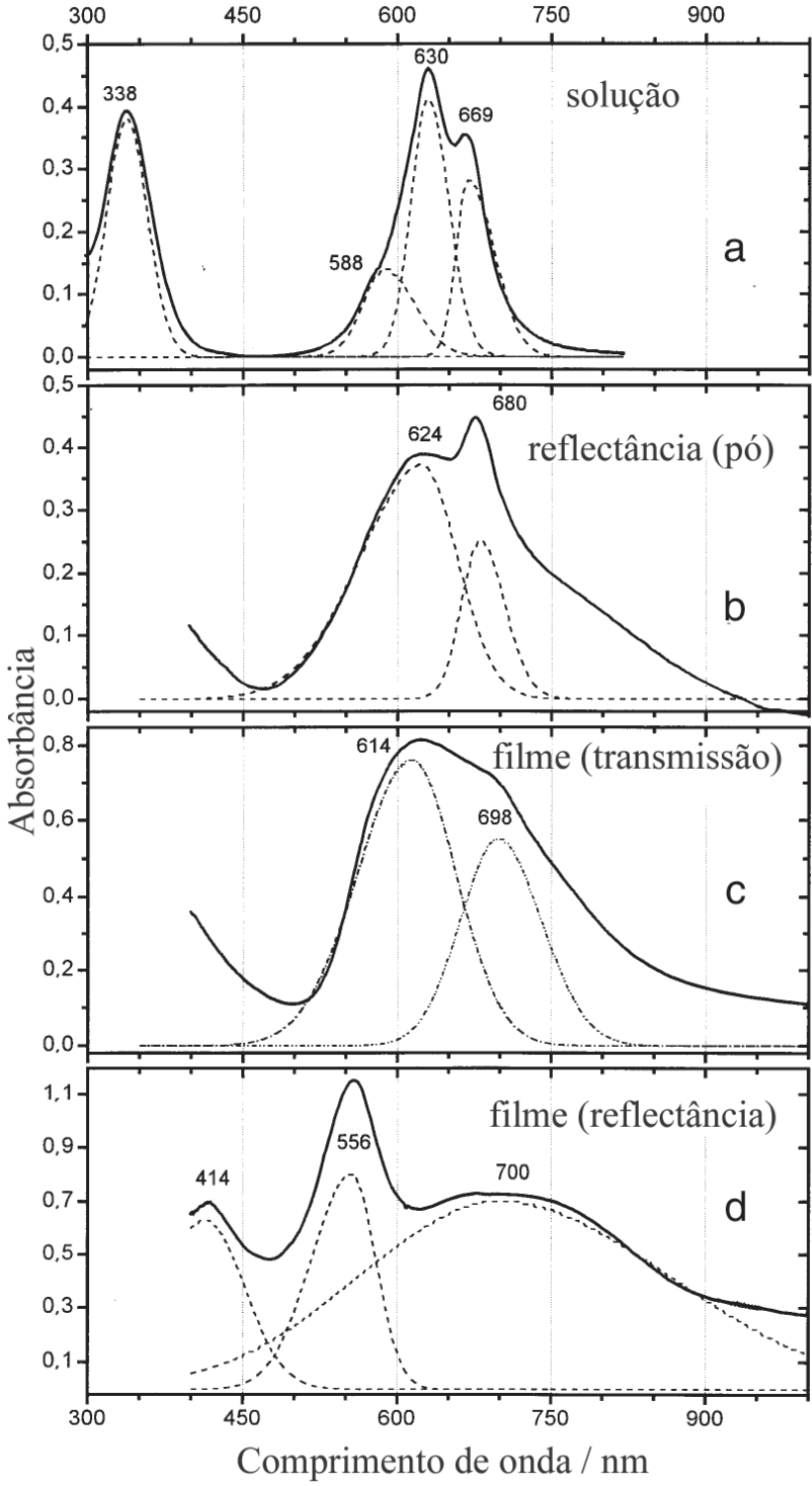

Figura 4. Espectros eletrônicos para a ftalocianina de cobre: a) solução aquosa $1 \times 10^{-4} \mathrm{~mol} \mathrm{dm}^{-3}$; b) pastilha (pó) - reflectância difusa; c) filme - por transmissão e d) filme - por reflectância especular (linha sólida - espectro experimental; linha pontilhada - deconvolução espectral)

\section{AS CORES DO OURO}

Enquanto o brilho dourado tem sua origem na reflexão especular, causa surpresa o fato que sob a forma de filmes suficientemente finos para transmitir a luz, o ouro se apresenta verde! Certamente, muitas das obras de arte com pinturas em ouro, têm seus complexos efeitos cromáticos provocados pela combinação de absorção e reflexão.

Mas o ouro é ainda capaz de assumir várias cores (Figura 5), quando sob a forma de nanopartículas ${ }^{5}$. Com dimensões entre 2 e $10 \mathrm{~nm}$, as nanopartículas de ouro apresentam intensa coloração vermelha, devido a transições entre as bandas eletrônicas que acomodam os elétrons superficiais (bandas de plasmons superficiais). Entretanto, em tamanhos maiores as nanopartículas passam a exibir uma coloração tendendo ao violeta, devido ao aumento na densidade de estados eletrônicos e conseqüente diminuição da energia de transição entre as bandas. Assim, as nanopartículas de ouro com 
diâmetros médio iguais a 9, 15, 22, 48 e 99 nm apresentam máximos de absorção em $517,520,521,533$ e 575 nm, respectivamente, em meio aquoso.

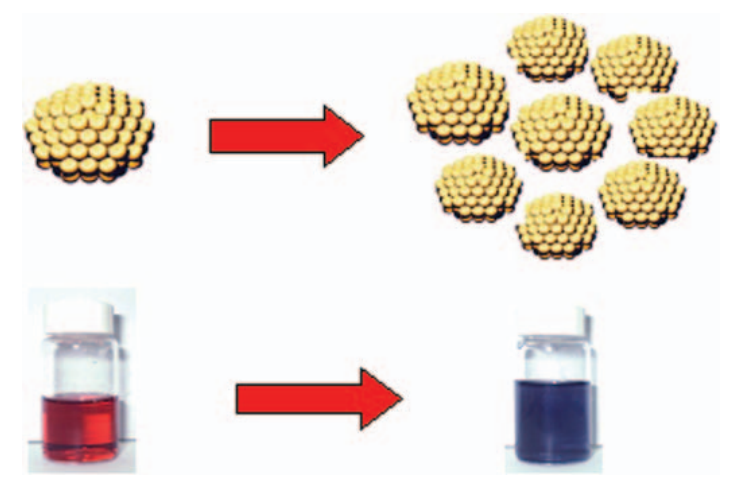

Figura 5. Mudança na coloração de uma suspensão de nanopartículas de ouro em água devido ao processo de agregação, na presença de 4mercaptopiridina. As imagens mostradas foram obtidas por fotografia digital

Um dos principais métodos para a síntese de nanopartículas de ouro, desenvolvido por Turkevitch et al. ${ }^{10}$, baseia-se na redução e estabilização das nanopartículas pelo íon citrato. Para que as nanopartículas de ouro se mantenham estáveis e dispersas em uma suspensão é essencial a presença de uma camada estabilizante, que evite a aglomeração, seja por repulsão eletrostática ou por impedimento estérico. A agregação é facilmente reconhecida pelo deslocamento da banda de plasmons para regiões de menor energia. No exemplo da Figura 6, a banda desloca-se para $750 \mathrm{~nm}$, gerando uma coloração azulada característica.

A Figura 6 exibe os espectros eletrônicos obtidos antes e após o processo de agregação de nanopartículas sintetizadas através do método de Turkevitch, mostrando a diminuição da energia de transição do plasmon superficial com o aumento do tamanho das nanopartículas. A agregação foi promovida através da adição de 4mercaptopiridina, que desloca a camada estabilizante de citrato, devido à alta afinidade do grupamento tiol pelo ouro. Por não possuir cargas, a 4-mercaptopiridina não é capaz de estabilizar as nanopartículas com eficiência, podendo ainda atuar como ponte entre as nanopartículas, através dos átomos de enxofre e nitrogênio ${ }^{11}$.

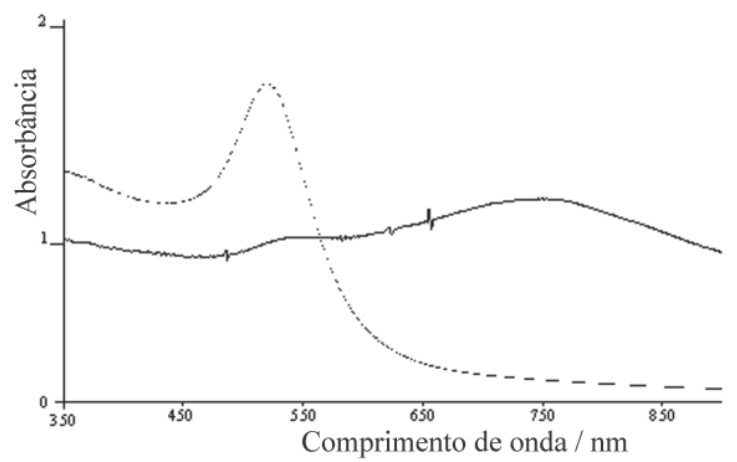

Figura 6. Espectros eletrônicos de suspensões de nanopartículas de ouro antes (linha pontilhada) e após (linha contínua) o processo de agregação (na presença de 4-mercaptopiridina $)^{I I}$

É interessante comparar as colorações de uma suspensão de nanopartículas de ouro em água (cor de vinho) e de um filme fino de ouro, depositado por termovaporização (verde). Nos espectros exibidos na Figura 7, nota-se que a banda de transição do plasmon em $520 \mathrm{~nm}$ deixa de ser observada quando se tira o espectro do filme de ouro, onde predomina o mecanismo de reflexão especular, gerando um mínimo de absorção nessa região. É curioso, que nesse comprimento de onda de absorção mínima, a absortividade seja na realidade máxima!

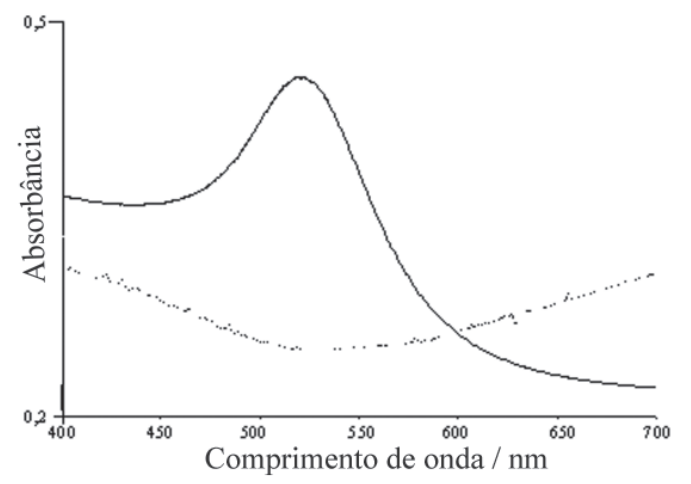

Figura 7. Espectros eletrônicos de nanopartículas de ouro em suspensão aquosa (linha contínua) e de um filme das mesmas (linha tracejada)

\section{CONSIDERAÇÕES FINAIS}

$\mathrm{Na}$ arte e na ciência, as cores e os espectros misturam-se, criando múltiplas visões, artefatos e contrastes. A física da cor, através dos efeitos cromáticos da refração e reflexão da luz, não apenas ilude os nossos olhos, como também consegue enganar os equipamentos, gerando bandas e transições inexistentes, por ex., nos filmes refletivos de ftalocianinas de cobre. Na escala nanométrica, a dimensão das partículas também influi nos efeitos de cor, criando oportunidades para aplicações em sensores e até em computação quântica ${ }^{12}$. Longe de esgotar o assunto, estendemos ao leitor o convite para enveredar pela arte de Israel Pedrosa e descobrir a beleza e os desafios da cor inexistente.

\section{AGRADECIMENTOS}

À FAPESP, CNPq, RENAMI, IM2C e ao CRQ-4, pelo apoio.

\section{REFERÊNCIAS}

1. Pedrosa, I.; Da cor à cor inexistente, 3a. ed., Ed. Léo Christiano, Co-editora Univ. Brasília: Rio de Janeiro, 1982.

2. Klintowitz, J.; O ofício da arte: a pintura, Ed. Abram Szajman: São Paulo, SESC, 1987.

3. http://www.cameraweb.unicamp.br/acervo/acervo2.html, acessada em Junho 2004.

4. Leznoff, C. C.; Lever, A. B. P.; Phtalocyanines: Properties and Applications, VCH: New York, 1989, vol. 1.

5. Daniel, M.-C.; Astruc, D.; Chem. Rev. 2004, 104, 293.

6. Duff, J. A.; Bonding, energy levels and bands in inorganic solids, Longman, Essex: Inglaterra, 1990.

7. Marder, S. R. Em Inorganic Materials; Bruce, D. W.; O’Hare D., eds.; John Wiley \& Sons: Chichester, 1992, cap. 3.

8. Kasha, M.; Rawls, H. R.; El-Bayoumi, M. A.; Pure Appl. Chem. 1965, 11, 371.

9. Wright, J. D.; Molecular Crystal, Cambridge University Press: Cambridge, 1995.

10. Turkevitch J.; Stevenson, P. C.; Hilier J.; Discuss. Faraday Soc. 1951, 11, 55.

11. Bonifácio, L. S.; Desenvolvimento de nanopartículas de ouro estabilizadas com sistemas metal-orgânicos visando aplicações em nanotecnologia, Monografia, sob orientação de Toma, H. E.; premiada pelo Conselho Regional de Química, 4a Região, 2004.

12. Toma, H. E.; O mundo nanométrico: a dimensão do novo século, Ed. Oficina de Textos: São PAulo, 2004. 